OPEN

SUBJECT AREAS:

SYNTHESIS AND

PROCESSING

CHEMICAL PHYSICS

NANOPARTICLES

POLLUTION REMEDIATION

Received

22 October 2014

Accepted

16 February 2015

Published

12 March 2015

Correspondence and requests for materials should be addressed to G.W.Y. (stsygw@mail. sysu.edu.cn)

\section{Super adsorption capability from amorphousization of metal oxide nanoparticles for dye removal}

\author{
L. H. Li, J. Xiao, P. Liu \& G. W. Yang
}

State Key Laboratory of Optoelectronic Materials and Technologies, Nanotechnology Research Center, School of Physics \& Engineering, Sun Yat-sen University, Guangzhou 510275, Guangdong, P. R. China.

Transitional metal oxide nanoparticles as advanced environment and energy materials require very well absorption performance to apply in practice. Although most metal oxides are based on crystalline, high activities can also be achieved with amorphous phases. Here, we reported the adsorption behavior and mechanism of methyl blue (MB) on the amorphous transitional metal oxide (Fe, Co and Ni oxides) nanoparticles, and we demonstrated that the amorphousization of transitional metal oxide (Fe, $\mathrm{Co}$ and $\mathrm{Ni}$ oxides) nanoparticles driven by a novel process involving laser irradiation in liquid can create a super adsorption capability for $\mathrm{MB}$, and the maximum adsorption capacity of the fabricated $\mathrm{NiO}$ amorphous nanostructure reaches up to $10584.6 \mathrm{mgg}^{-1}$, the largest value reported to date for all $\mathrm{MB}$ adsorbents. The proof-of-principle investigation of $\mathrm{NiO}$ amorphous nanophase demonstrated the broad applicability of this methodology for obtaining new super dyes adsorbents.

T ransitional metal oxide nanoparticles have been studied extensively due to their excellent performances as advanced nanomaterials in areas of environment and energy, and adsorption plays a crucial role in these applications, e.g., strong adsorbing can greatly improve performance ${ }^{1-9}$. Therefore, enhancing adsorption of metal oxide nanoparticles has been a subject that has fascinated scientists and engineers for several decades. Wastewater from textile, pharmaceutical and food processing industry have always been a serious environmental problem, which are harmful to aquatic life and even endanger human health. Many dyes as organic contaminants are usually stable and hardly degradated in conventional wastewater treatment techniques including flocculation, coagulation, chemical precipitation and biological oxidation ${ }^{10,11}$. Developing environment friendly adsorption techniques is thus becoming an important issue to remove organic contaminants in water via simply adsorbing $^{12,13}$. Methyl blue (MB) is one of the most common dyes which is widely used as the coloring agent and disinfector in pesticides, pharmaceuticals, dyestuffs and varnishes ${ }^{14-16}$, and it is generally adopted as a representative organic pollutant to test the adsorption performance for the removal of organic contaminants from wastewater. However, up to date, the maximum adsorption capacity of all the reported MB adsorbents is still below $10^{4} \mathrm{mgg}^{-1}$ (Supplementary Information S1).

Here, we synthesize amorphous transitional metal oxide ( $\mathrm{Fe}$, Co and Ni oxides) nanoparticles by a simple and green process, i.e., laser irradiation in liquid. Our measurements indicate that the maximum adsorption capacity of the fabricated amorphous nanostructures of metal oxide nanoparticles for $\mathrm{MB}$ is beyond $10^{4} \mathrm{mgg}^{-1}$ and high up to $10584.6 \mathrm{mgg}^{-1}$, which is the largest value reported to date for all MB adsorbents. These findings thus promote the application of amorphous nanostructures as advanced adsorbent materials.

\section{Results}

Morphology and structural characterizations. The morphology and structure evolution of the starting metal oxides nanoparticles upon the laser irradiation in liquid is shown in Fig. 1. From these scanning electron microscopy (SEM) and Transmission electron microscopy (TEM) images, we can see the evolution that the particles are becoming smaller. The final products are the amorphous nanostructures as shown in the selected area electron diffraction (SAED) image insets in Fig. 1. Meanwhile, the corresponding X-ray diffraction (XRD) patterns reveal the detailed structural evolution of the starting raw materials from crystal to amorphous (Supplementary Information S2). Therefore, these results show that the amorphization of transitional metal 

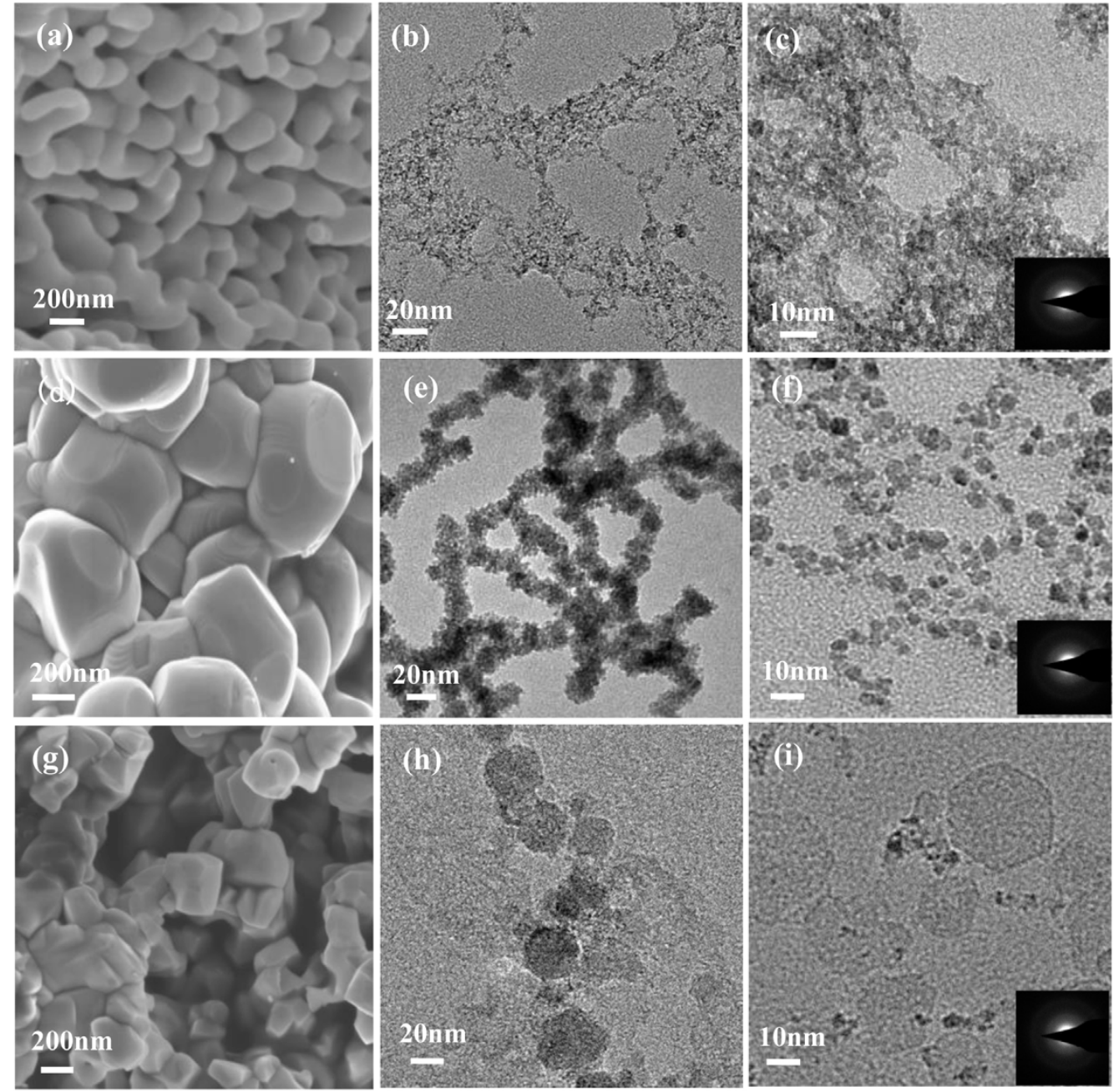

Figure 1 The morphology and structure evolution of the starting metal oxides nanoparticles upon the laser irradiation in liquid. (a), (d) and (g) TEM iamges of the starting nanoparticles of $\mathrm{Fe}_{2} \mathrm{O}_{3}, \mathrm{CoO}$ and $\mathrm{NiO}$, respectively. (b-c), (e-f) and (h-i) TEM images of the synthesized products of $\mathrm{Fe}_{2} \mathrm{O}_{3}, \mathrm{CoO}$ and $\mathrm{NiO}$, respectively. The insets in (c), (f) and (i) are the corresponding $\mathrm{SAED}$ patterns of the $\mathrm{Fe}_{2} \mathrm{O}_{3}, \mathrm{CoO}$ and $\mathrm{NiO}$ samples, respectively.

oxides nanoparticles driven by the laser irradiation in liquid is achieved. Note that the Brunauer-Emmett-Teller (BET) surface areas of the synthesized products are promoted to be larger 10-30 times than that of the starting nanoparticles (Supplementary Information S3).

Effects of $\mathbf{p H}$ on adsorption. It is well known that the effect of $\mathrm{pH}$ plays a significant role on the active sites of nano-adsorbents as well as the dye species during the adsorption reaction. The experiments are carried out in $\mathrm{pH}$ range 2.0-11.8 and the results are illustrated in Fig. 2. The chart clearly reveals that the removal degree of MB increases as the $\mathrm{pH}$ value rises from 2 to 6.5 , and then decreases rapidly at higher $\mathrm{pH}$ value. The optimum $\mathrm{pH}$ for the samples is 6.5. At acidic $\mathrm{pH}$, it is obviously that the adsorption capacity of $\mathrm{MB}$ decreased at low $\mathrm{pH}$ is explained by the fact that the leaching of $\mathrm{Fe}, \mathrm{Co}, \mathrm{Ni}$, which might destroy the structure of the samples. At alkaline $\mathrm{pH}$, decreasing $\mathrm{MB}$ adsorption at high $\mathrm{pH}$ may be due to the competition of $\mathrm{OH}$ with $\mathrm{MB}$ ions for the adsorption sites on the samples. The increasing number of hydroxyl groups decreases the number of positively charged sites and reduces the attraction between dye and adsorbent surface. Therefore, the possible mechanism of $\mathrm{MB}$ adsorption is considered as the strong electrostatic interaction between the positive active site of the adsorbent and the negative charge of MB.
Adsorption isotherm. To comprehend the adsorption potential between adsorbent and adsorbate, the working adsorption isotherm modes such as Langmuir, Freundlich and Temkin need to be determined (Supplementary Information S4). The adsorption experiments are conducted at $298 \mathrm{~K}$ and $\mathrm{pH}=6.5$. The adsorption isotherms are illustrated in Fig. 3. From these data, we can see that, the $\mathrm{CoO}$ and $\mathrm{NiO}$ samples work in the Temkin mode, and the $\mathrm{Fe}_{2} \mathrm{O}_{3}$ sample works in the Freundlich mode. Meanwhile, we can see that the adsorption capacity of the fabricated colloidal solution is promoted to be higher 20 to 30 times than that of the starting raw materials. Importantly, the maximum MB adsorption capacity of the synthesized $\mathrm{NiO}$ colloidal solution is beyond absorption limit of $10^{4} \mathrm{mgg}^{-1}$ and high up to $10584.6 \mathrm{mgg}^{-1}$, which is the largest value reported to date for all $\mathrm{MB}$ adsorbents.

Adsorption kinetics. The adsorption kinetics of MB onto amorphous nanostructures of metal oxides nanoparticles are evaluated by utilizing the pseudo first-order and pseudo second-order models (Supplementary Information S5). These results (Fig. 4a-c) show that the pseudo-second-order kinetic model fits well the adsorption kinetics of $\mathrm{MB}$ onto the samples in our case, and the rate limiting step would be chemisorptions ${ }^{17}$. Clearly, we can see that $\mathrm{MB}$ can be removed $99.0 \%$ via the $\mathrm{NiO}$ sample in $1 \mathrm{~min}$., while $90.3 \%$ via the starting raw nanoparticles in $150 \mathrm{~min}$. 

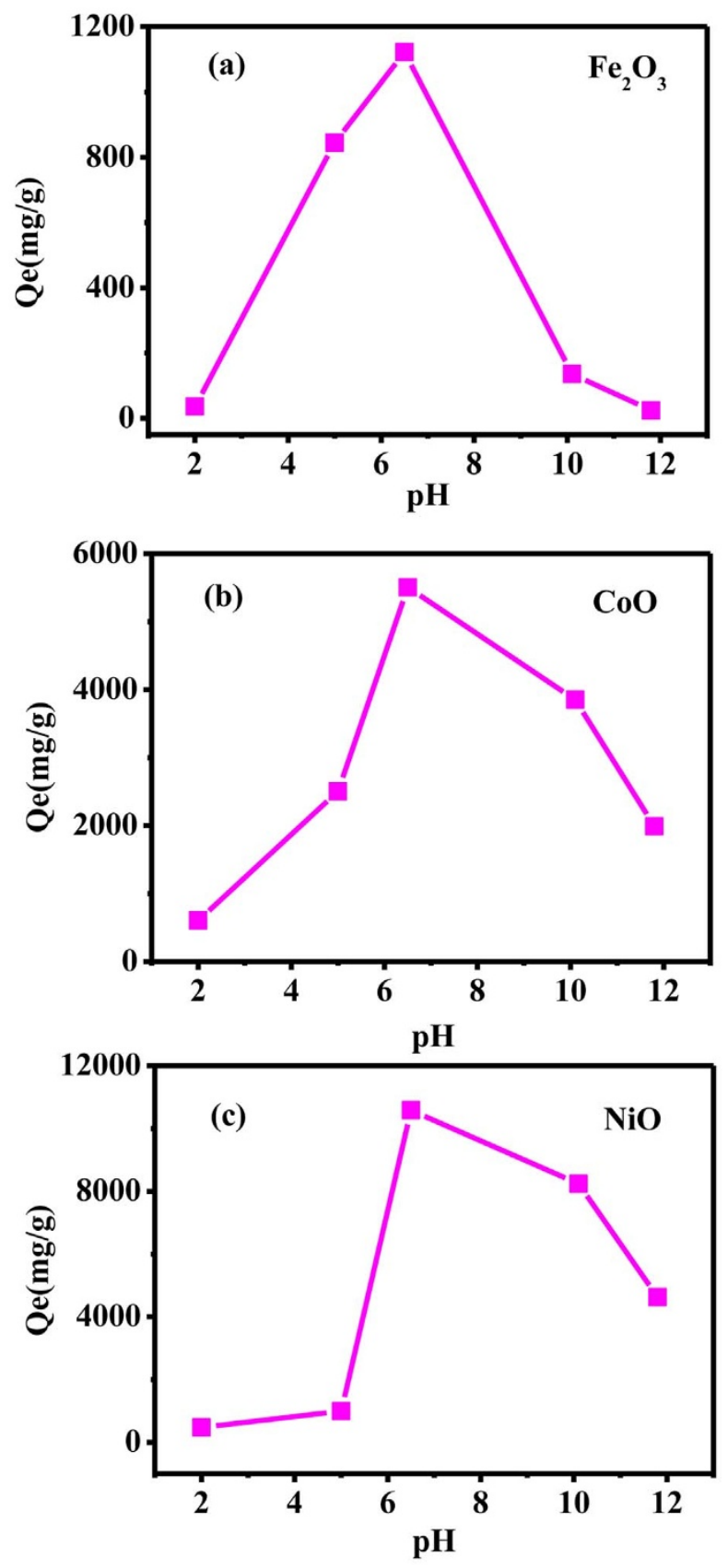

Figure $2 \mid$ The effect of initial $\mathrm{pH}$ on adsorption. (a) $\mathrm{Fe}_{2} \mathrm{O}_{3}$, (b) $\mathrm{CoO}$ and (c) $\mathrm{NiO}$.

\section{Discussion}

Mechanism of MB adsorption onto amorphous transitional metal oxides. The super adsorption capacity induced by the amorphization of transitional metal oxides nanoparticles is attributed to these reasons below. Firstly, from Supplementary Information S3, we can clearly see that the BET surface areas of the synthesized products are larger $10-30$ times than that of the starting nanoparticles. Thus, larger surface area created by the amorphization of transitional metal oxide nanoparticles is very beneficial to the adsorption performance of the synthesized products.

Secondly, we systemically compare the surface states of the starting raw materials and the synthesized colloidal solutions, and that of the synthesized materials before and after adsorbing MB by Fourier Transform Infrared Spectroscopy (FTIR) as shown in Fig. 5 and Supplementary Information S6. Clearly, we can see that the FTIR spectra of the starting raw materials has not basically the adsorption peaks, but a lot of $\mathrm{OH}$ radicals have been generated in the synthesized colloidal solutions. After adsorbing $\mathrm{MB}$, the aromatic ring vibrations at 1575,1495 and $1449 \mathrm{~cm}^{-1}$, the C-N stretching vibration at $1337 \mathrm{~cm}^{-1}$ and the $\mathrm{SO}_{3} \mathrm{Na}$ radical vibrations at $1169,1121,1032$ and $1005 \mathrm{~cm}^{-1}$ emerge in the spectra of the fabricated colloidal solutions, which reflecting the evidence that chemical bonds are very strong in this case. Since the reaction ability of $\mathrm{OH}$ radicals is high enough to attack almost any organic molecules such as $\mathrm{SO}_{3} \mathrm{Na}$ radicals, it is assigned as a key species in the adsorption mechanism of many hazardous chemical compounds. Therefore, these results reveal the strong interaction between $\mathrm{MB}$ and amorphous nanostructures. Note that $\mathrm{M}(\mathrm{Fe}, \mathrm{Co}$ and $\mathrm{Ni})-\mathrm{O}$ bonds are the main vibrations in the low wave number. We can infer that between $\mathrm{M}^{2+}$ in $\mathrm{M}\left(\mathrm{OH}^{+}\right)$and $\mathrm{O}^{2-}$ in sulfonic groups in $\mathrm{MB}$ exits strong ionic bonding. The amorphization of transitional metal oxides nanoparticles provides much more active sites as adsorption sites and hangs more function groups which greatly promote the adsorbing performance.

Thirdly, the surface charge of the synthesized colloidal solutions plays a key role in the $\mathrm{MB}$ adsorption. Considering the $\mathrm{pHpzc}$ of the amorphous transitional metal oxide ( $\mathrm{Fe}, \mathrm{Co}$ and $\mathrm{Ni}$ oxides) nanoparticles, $\left(\mathrm{pHpzc}=8.7,10.5,10.7\right.$ for $\mathrm{Fe}_{2} \mathrm{O}_{3}, \mathrm{CoO}, \mathrm{NiO}$ respectively) the surface formation of $\mathrm{M}(\mathrm{Fe}, \mathrm{Co}$ and $\mathrm{Ni}) \mathrm{O}$ is mainly $\mathrm{M}\left(\mathrm{OH}^{+}\right)$at $\mathrm{pH}=6.5$ in our experiments (Supplementary Information S7). Hence, the interaction between $\mathrm{MB}$ and $\mathrm{M}(\mathrm{Fe}, \mathrm{Co}$ and $\mathrm{Ni}) \mathrm{O}$ might be mainly the ionic bonding between the positively charged center of $\mathrm{M}(\mathrm{Fe}, \mathrm{Co}$ and $\mathrm{Ni})\left(\mathrm{OH}^{+}\right)$and negatively charged functional groups of $\mathrm{MB}\left(-\mathrm{SO}_{3}{ }^{-}\right)$. Thus, this strong interaction also improves adsorption. Additionally, the zeta potential values (Supplementary Information S7) verify the deduction above.

Transformation mechanism of crystals nanoparticles into amorphous ones. The reasons that the transformation from crystals nanoparticles into amorphous ones under laser irradiation in liquid can be ascribed to two aspects below. For one thing, the laser energy with larger power density ( $400 \mathrm{~mJ} /$ pulse in our case) is sufficient to destroy the order crystalline structure. Compared with crystal, amorphous usually keeps the larger cohesive energy, which means that they are unstable state that can be synthesized in the nonequilibrium condition created by laser ablation in liquid. For another, water environment may be essential for the formation of amorphous. Khan et. al. claimed that they used laser to ablate Ni plate in water to produce amorphous $\mathrm{NiO} \mathrm{NPs}{ }^{14}$. Also, Shim and his co-worker used laser to ablate $\mathrm{Fe}_{2} \mathrm{O}_{3}$ target in water and obtain amorphous $\mathrm{Fe}_{2} \mathrm{O}_{3}$. However, they got crystal when they choose alcohol and acetone as liquid ${ }^{15}$. Therefore, these evidences indicate that the water may easily form amorphous layer or benefit for the generation of amorphous for metal oxides.

Capacity comparison and selectivity of MB adsorption onto amorphous transitional metal oxides. We compare the adsorption performances of the synthesized colloidal solutions for $\mathrm{MB}$, methylene blue (YMB) and methyl orange (MO) (Supplementary Information S8). Definitely, these colloidal solutions show relatively poor performances in the processes of $\mathrm{YMB}$ and $\mathrm{MO}$ adsorption, which is attributed to the absence of $\mathrm{SO}_{3} \mathrm{Na}$ function groups in $\mathrm{YMB}$ and $\mathrm{MO} . \mathrm{MB}, \mathrm{MO}$ and $\mathrm{YMB}$ have three, one and zero $\mathrm{SO}_{3} \mathrm{Na}$, respectively. Therefore, the adsorption of the synthesized colloidal solutions is highly selective.

In summary, a series of the amorphization of transitional metal oxide nanoparticles have been achieved and the related amorphous nanostructures as super adsorption materials have been fabricated by a simple and environmentally friendly laser irradiation in liquid. The synthesized colloidal solution of amorphous $\mathrm{NiO}$ performed a super adsorption capacity for $\mathrm{MB}$, especially the maximum $\mathrm{MB}$ adsorption capacity is beyond absorption limit of $10^{4} \mathrm{mgg}^{-1}$ and reaches up to $10584.6 \mathrm{mgg}^{-1}$, which is the largest value reported to date for MB 

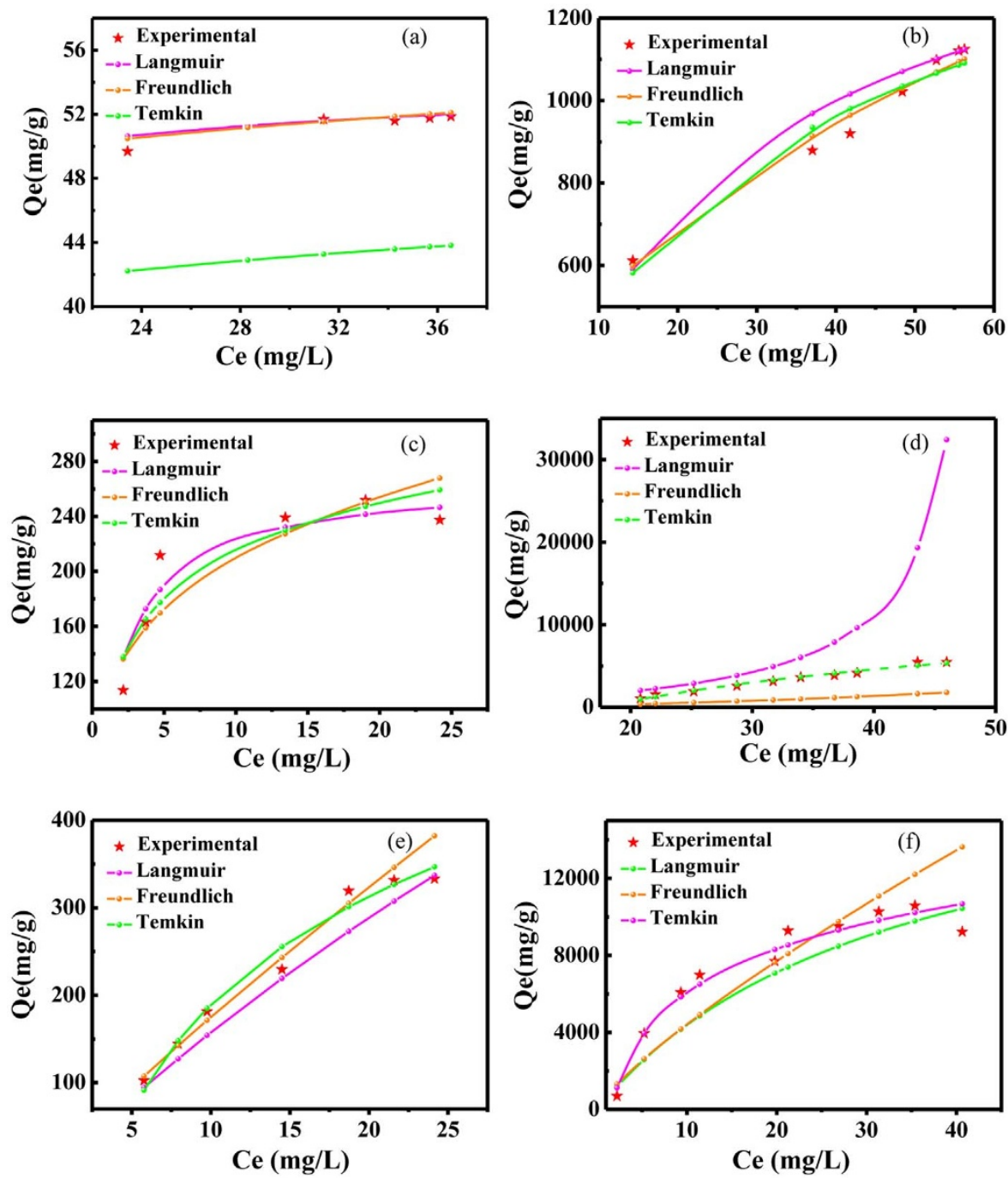

Figure 3 Comparison of the equilibrium isotherms between the experimental and theoretical data. (a-c) The starting nanoparticles of $\mathrm{Fe}_{2} \mathrm{O}_{3}$, $\mathrm{CoO}$ and $\mathrm{NiO}$, respectively. (d-f) The fabricated amorphous nanostructures of $\mathrm{Fe}_{2} \mathrm{O}_{3}, \mathrm{CoO}$ and $\mathrm{NiO}$, respectively.
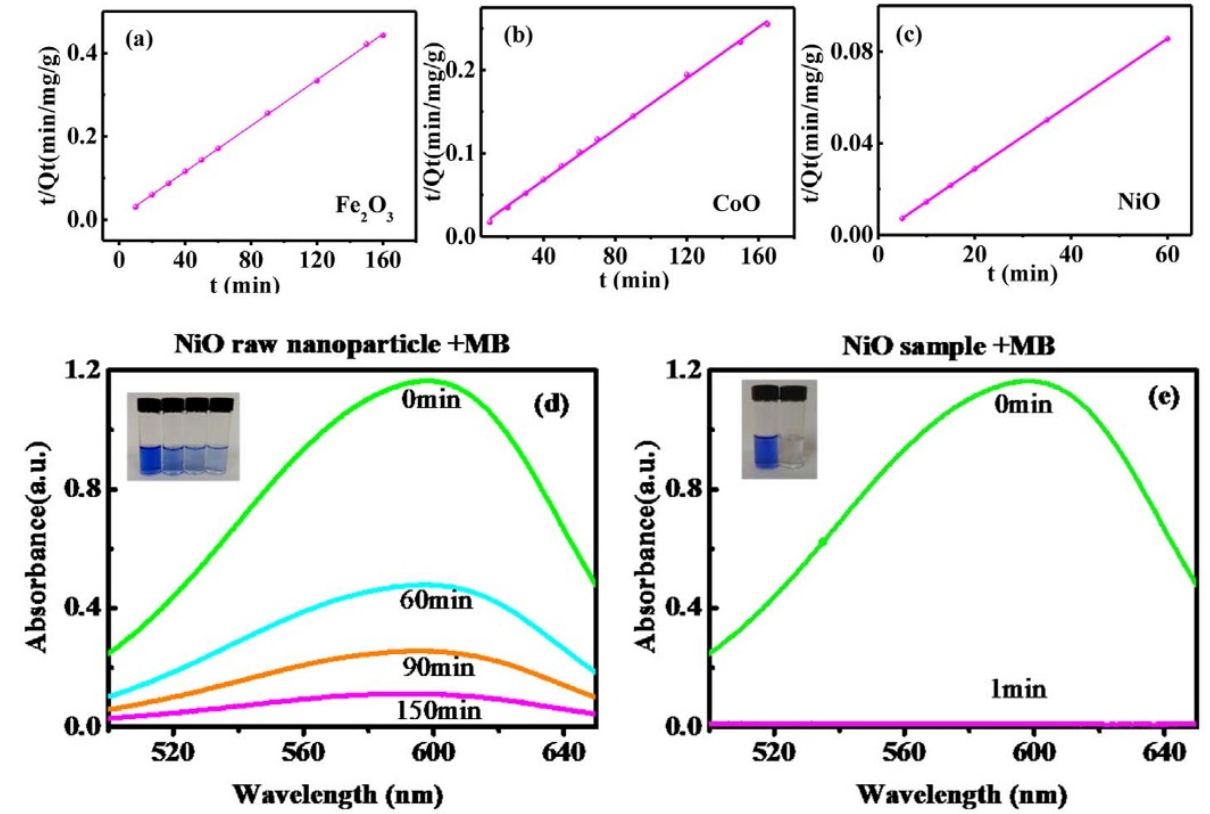

Figure $4 \mid$ Adsorption kinetic results. (a-c) All three samples exhibt the pseudo second-order kinetic behaviors. The adsorbing rates of the starting NiO nanoparticles (d) and the synthesized amorphous nanostructures (e). 

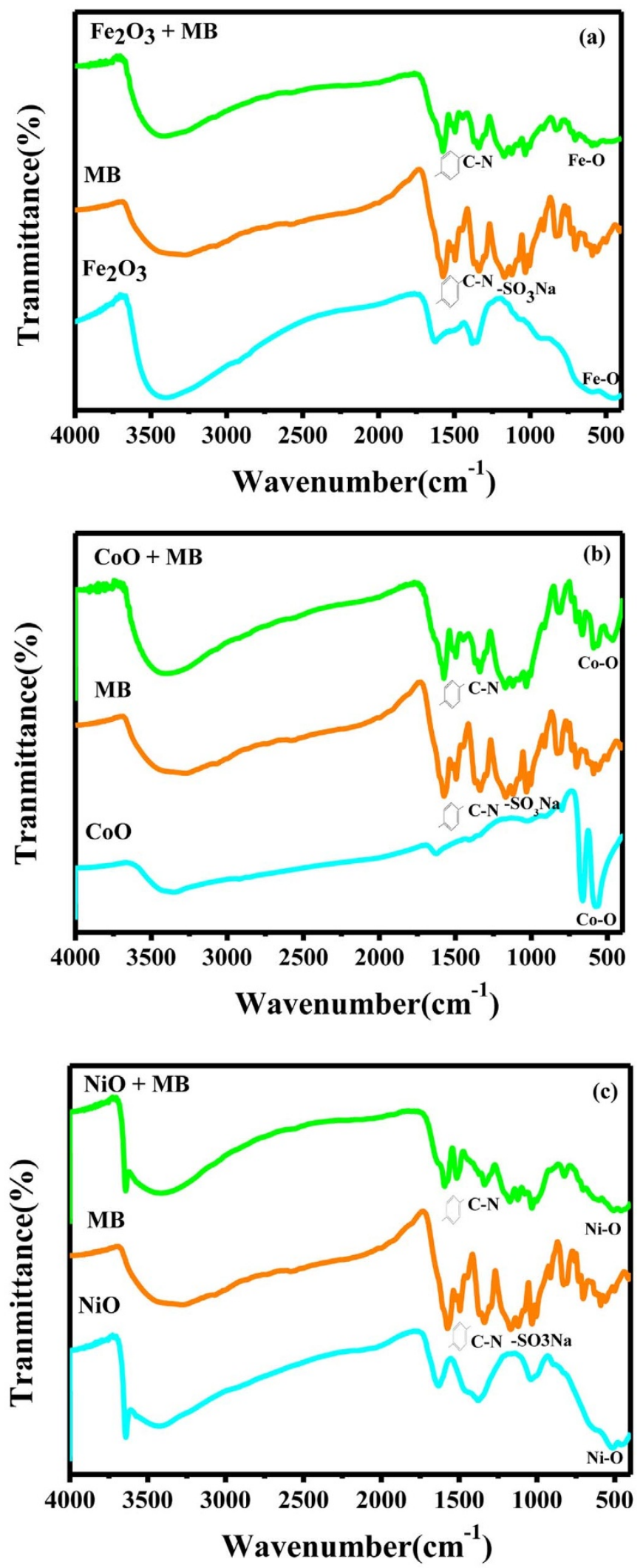

Figure 5 FTIR spectra of the fabicated colloidal solutions, the MB solution and the colloidal solutions adsorbed by $\mathrm{MB}$. (a) $\mathrm{Fe}_{2} \mathrm{O}_{3}$, (b) $\mathrm{CoO}$ and (c) NiO.

adsorbents. $\mathrm{MB}$ can be removed $99.0 \%$ via amorphous $\mathrm{NiO}$ in $1 \mathrm{~min}$, while $90.3 \%$ via the starting raw nanoparticles in $150 \mathrm{~min}$. Therefore, these findings demonstrated the broad applicability of this methodology for accessing new super dyes adsorbents.

\section{Methods}

Materials synthesis. Iron (III) oxide, Nickel (II) oxide, Cobalt (II) oxide, Methyl Orange and Methylene blue are from Alfa Aesar (China), and Methyl blue is from Sigma-Aldrich (China). All chemicals are of analytical grade. All solutions are prepared with deionized water. The laser irradiation in liquid technique has been reported in our previous works ${ }^{18-26}$. In this case, $5 \mathrm{mg}$ metal oxide nanoparticles powder with a purity of $99.99 \%$ is firstly placed in a glass bottle filled with $10 \mathrm{ml}$ of deionized water. Magnetically-stirred operation is to make the powder monodisperse in water. Then, the aqueous solution is irradiated by a Q-switched Nd:YAG laser device with a wavelength of $532 \mathrm{~nm}$, repeating frequency of $10 \mathrm{~Hz}$, pulse width of $10 \mathrm{~ns}$, energy density of $400 \mathrm{~mJ} / \mathrm{pulse}$ and spot size of $4 \mathrm{~mm}$ in diameter. During laser irradiating, the liquid environment is maintained at ambient temperature and pressure. The laser irradiation process lasts for $30 \mathrm{~min}$. Then, the colloidal solution is synthesized and then collected in a cuvette for measurements.

Materials characterization. The materials are characterized by SEM with Thermal Field Emission Environmental SemEdsEBSD. XRD is performed with a Rigaku Dmax 2200 VPC (Japan) with $\mathrm{Cu} \mathrm{K} \alpha$ radiation $(\lambda=1.54056 \AA$, accelerating voltage is $40 \mathrm{kV}$, emission current is $26 \mathrm{~mA}$ ), and a scanning rate of $1^{\circ} \mathrm{s}^{-1}$ is employed. TEM is carried out with a JEOL JEM-2010HR instrument (Japan) at an accelerating voltage of $200 \mathrm{kV}$. The samples are ultrasounded for 30 minutes and then dropped pipette onto a ultrathin carbon supported film. Above mentioned techniques are used to identify morphology, structure and composition of the as-synthesized samples. The optical absorption of the colloidal dispersion is measured by using the UV-Vis-NIR 3150 Spectrophotometer (Japan). The nitrogen adsorption-desorption isotherms are measured by Automated gas sorption analyzer Autosorb-iQ2-MP (USA) at $77 \mathrm{~K}$. And the specific surface area of the materials is calculated by the Brunauer-EmmettTeller (BET) theory. The Zeta potential is carried out by Nanoparticle size-Zeta potential and molecular weight analyzer (Brookhaven). The Inductively coupled plasma-atomic emission spectrometry (ICP-AES) using a ThermoFisher Icap6500Duo is employed to analyze the concentration of $\mathrm{Fe}, \mathrm{Co}$, $\mathrm{Ni}$, with an incident power of $1150 \mathrm{~W}$, a plasma gas flow of $14 \mathrm{~L} / \mathrm{min}$, and an atomization gas flow of $0.6 \mathrm{~L} / \mathrm{min}$. FTIR of the samples are recorded with the Fourier transformation infrared spectrometer coupled with infra-red microscope (EQUINOX 55).

Effects of $\mathbf{p H}$ on adsorption. In order to evaluate the influence of $\mathrm{pH}$ on the adsorption capacity of the samples, experiments are carried out at initial concentration of $60 \mathrm{mg} / \mathrm{L}(\mathrm{pH}>7)$ and $6 \mathrm{mg} / \mathrm{L}(\mathrm{pH}<7)$ and in the range of 2.0 11.8 , the equilibrium time of $12 \mathrm{~h}$ at room temperature. The initial $\mathrm{pH}$ of the solutions is adjusted with $0.5 \mathrm{~mol} / \mathrm{L} \mathrm{HCl}$ and $0.5 \mathrm{~mol} / \mathrm{L} \mathrm{NaOH}$ solutions.

Adsorption kinetic experiments. All adsorption kinetic experiments are performed at ambient temperature and pressure. The adsorption isotherm is studied by adding $1 \mathrm{ml}$ of $415 \mathrm{mg} / \mathrm{L}$ fresh fabricated colloid solution $\left(1 \mathrm{ml}\right.$ of $350 \mathrm{mg} / \mathrm{L}$ for $\mathrm{Fe}_{2} \mathrm{O}_{3}$ and $\mathrm{CoO}$ colloid solution) to different volume (5-200 ml) of $60 \mathrm{mg} / \mathrm{L} \mathrm{MB}$ aqueous solution. Then, the solution is continuously stirred in dark at a constant speed of $150 \mathrm{rpm}$ for $12 \mathrm{~h}$ to ensure the established of an adsorption-desorption equilibrium among the sample, $\mathrm{MB}$, and water. Then, aliquots $(5.0 \mathrm{ml})$ of the samples are collected, centrifuged (centrifugation speed is $13500 \mathrm{rpm}$ for 10 minutes), filtered (Millipore, $0.22 \mu \mathrm{m}$ ). The kinetics (time ranging from 0-60 min for the $\mathrm{NiO}$ sample, time ranging from $0-165$ min for the $\mathrm{Fe}_{2} \mathrm{O}_{3}$ and $\mathrm{CoO}$ sample) is studied by adding $30 \mathrm{ml}$ of $415 \mathrm{mg} / \mathrm{L}$ fresh fabricated $\mathrm{NiO}$ colloid solution $\left(350 \mathrm{mg} / \mathrm{L}\right.$ for $\mathrm{Fe}_{2} \mathrm{O}_{3}$ and $\mathrm{CoO}$ colloid solution) to $150 \mathrm{ml} 60 \mathrm{mg} / \mathrm{L} \mathrm{MB}$ aqueous solution. Then, morsels $(5.0 \mathrm{ml})$ of samples are collected, centrifuged immediately to separate the suspended solid and filtered (Millipore, $0.22 \mu \mathrm{m}$ ) for further research at suitable time intervals. The determination are carried out by logging the maximum absorption using a UVvis spectrometer at $\lambda_{\max }=595 \mathrm{~nm}(\mathrm{pH}=6.5)$. All experiments are performed in duplicate. In order to judge the adsorption performance of the as-synthesized samples, the raw powders (the starting nanoparticles) and commercial activated carbon are introduced for comparison. The concentration reserved in the adsorbent phase $\left(Q_{e}, \mathrm{mg} / \mathrm{g}\right)$ is measured by using the following equation

$$
Q_{e}=\frac{\left(C_{o}-C_{e}\right) V}{m}
$$

where $C_{e}(\mathrm{mg} / \mathrm{L})$ is the initial dye concentration, $C_{t}(\mathrm{mg} / \mathrm{L})$ is the equilibrium dye concentration in the aqueous solution, $V(\mathrm{~L})$ is the volume of solution and $m(\mathrm{~g})$ is the mass of the adsorbent. The adsorption capacity of the A-MONP at time $t, Q_{t}(\mathrm{mg} / \mathrm{g})$ was also calculated

$$
Q_{t}=\frac{\left(C_{o}-C_{t}\right) V}{m}
$$

where $C_{t}$ is the concentration of MB at time $t(\mathrm{mg} / \mathrm{L})$.

1. Batzill, M. The surface science of graphene: Metal interfaces, CVD synthesis, nanoribbons, chemical modifications, and defects. Surf. Sci. Rep. 67, 83-115 (2012).

2. Chen, B. et al. Surface Interactions and Quantum Kinetic Molecular Sieving for $\mathrm{H}_{2}$ and $\mathrm{D}_{2}$ Adsorption on a Mixed Metal-Organic Framework Material. J. Am. Chem. Soc. 130, 6411-6423 (2008). 
3. Diebold, U. The surface science of titanium dioxide. Surf. Sci. Rep. 48, 53-229 (2003).

4. Furimsky, E. Hydrodenitrogenation of petroleum. Catal. Rev. Sci. Eng. 47, 297-489 (2005).

5. Furukawa, H., Cordova, K. E., O'Keeffe, M. \& Yaghi, O. M. The Chemistry and Applications of Metal-Organic Frameworks. Science 341, 1230444-1230456 (2013).

6. Rosenholm, J. M., Sahlgren, C. \& Linden, M. Towards multifunctional, targeted drug delivery systems using mesoporous silica nanoparticles - opportunities \& challenges. Nanoscale 2, 1870-1883 (2010).

7. Sun, C. H., Liu, L. M., Selloni, A., Lu, G. Q. \& Smith, S. C. Titania-water interactions: a review of theoretical studies. J. Mater. Chem. 20, 10319-10334 (2010).

8. Van Santen, R. A., Ghouri, M. M., Shetty, S. \& Hensen, E. M. H. Structure sensitivity of the Fischer-Tropsch reaction; molecular kinetics simulations. Catal. Sci. Technol. 1, 891-911 (2011).

9. Zimmermann, R., Freudenberg, U., Schweiss, R., Kuttner, D. \& Werner, C. Hydroxide and hydronium ion adsorption - A survey. Curr. Opin. J. Colloid Interface Sci. 15, 196-202 (2010).

10. Ajii, Z. \& Ali, A. M. Adsorption of methyl violet and brilliant blue onto poly(vinyl alcohol) membranes grafted with $\mathrm{N}$-vinyl imidazole/acrylic acid. Nucl. Instrum. Methods Phys. Res. Sect. B. 265, 362-365 (2007).

11. Ofomaja, A. E. \& Ho, Y.-S. Effect of temperatures and $\mathrm{pH}$ on methyl violet biosorption by Mansonia wood sawdust. Bioresour Technol. 99, 5411-5417 (2008)

12. Meunier, B. Catalytic Degradation of Chlorinated Phenols. Science 296, 270-271 (2002).

13. Tao, X. et al. Efficient degradation of organic pollutants mediated by immobilized iron tetrasulfophthalocyanine under visible light irradiation. Chem. Commun. 80-81; DOI:10.1039/B209083J (2003).

14. Khan, S. Z. et al. Generation and characterization of $\mathrm{NiO}$ nanoparticles by continuous wave fiber laser ablation in liquid. J. Nanopart. Res. 11, 1421-1427(2009).

15. Khai, T. V. et al. Synthesis of phase-controlled iron oxide nanoparticles by pulsed laser ablation in different liquid media. Phys. Status. Solidi. A 210, 563-569 (2013)

16. Wu, T. et al. Adsorption characteristics of acrylonitrile, p-toluenesulfonic acid, 1naphthalenesulfonic acid and methyl blue on graphene in aqueous solutions. Chem. Eng. J. 173, 144-149 (2011).

17. Duran, C., Ozdes, D., Gundogdu, A. \& Senturk, H. B. Kinetics and Isotherm Analysis of Basic Dyes Adsorption onto Almond Shell (Prunus dulcis) as a Low Cost Adsorbent. J. Chem. Eng. Data. 56, 2136-2147 (2011).

18. Chen, X. Y., Cui, H., Liu, P. \& Yang, G. W. Double-Layer Hexagonal Fe Nanocrystals and Magnetism. Chem. Mater. 20, 2035-2038 (2008).

19. Liu, P., Cao, Y. L., Chen, X. Y. \& Yang, G. W. Trapping High-Pressure Nanophase of Ge upon Laser Ablation in Liquid. Cryst. Growth. Des. 9, 1390-1393 (2008).
20. Xiao, J. et al. A new insight into the electrochemical growth of Ag nanodendrites without a strong electrolyte. RSC Adv. 3, 20532 (2013).

21. Liu, P., Cao, Y. L., Wang, C. X., Chen, X. Y. \& Yang, G. W. Micro- and Nanocubes of Carbon with C8-like and Blue Luminescence. Nano Lett. 8, 2570-2575 (2008).

22. Xiao, J., Liu, P., Liang, Y., Li, H. B. \& Yang, G. W. Super-stable ultrafine betatungsten nanocrystals with metastable phase and related magnetism. Nanoscale 5 , 899-903 (2013).

23. Xiao, J., Liu, P., Liang, Y., Li, H. B. \& Yang, G. W. Super-stable ultrafine betatungsten nanocrystals with metastable phase and related magnetism. Nanoscale 5 , 899 (2013).

24. Yang, G. W. Laser ablation in liquids: Applications in the synthesis of nanocrystals. Prog. Mater. Sci. 52, 648-698 (2007)

25. Xiao, J., Ouyang, G., Liu, P., Wang, C. X. \& Yang, G. W. Reversible Nanodiamond Carbon Onion Phase Transformations. Nano Lett. 14, 3645-3652 (2014).

26. Xiao, J., Liu, P., Liang, Y., Li, H. B. \& Yang, G. W. High aspect ratio $\beta-\mathrm{MnO}_{2}$ nanowires and sensor performance for explosive gases. J. Appl. Phy. 114, 073513 (2013)

\section{Acknowledgments}

The National Basic Research Program of China (2014CB931700) and the State Key Laboratory of Optoelectronic Materials and Technologies of Sun Yat-sen University supported this work.

\section{Author contributions}

G.W.Y. designed the experiments; L.H.L. and J.X. carried out the experiments; P.L. and J.X. carried out data analysis; L.H.L. and G.W.Y. wrote the paper.

\section{Additional information}

Supplementary information accompanies this paper at http://www.nature.com/ scientificreports

Competing financial interests: The authors declare no competing financial interests.

How to cite this article: Li, L.H., Xiao, J., Liu, P. \& Yang, G.W. Super adsorption capability from amorphousization of metal oxide nanoparticles for dye removal. Sci. Rep. 5, 9028; DOI:10.1038/srep09028 (2015)

This work is licensed under a Creative Commons Attribution 4.0 International License. The images or other third party material in this article are included in the article's Creative Commons license, unless indicated otherwise in the credit line; if the material is not included under the Creative Commons license, users will need to obtain permission from the license holder in order to reproduce the material. To view a copy of this license, visit http://creativecommons.org/licenses/by/4.0/ 